\title{
Síntese de magnesioferrita a partir de magnetitas magnesianas
}

\section{Magnesioferrite synthesized from magnesian-magnetites}

\author{
Marcelo Hidemassa Anami ${ }^{1}$; Antonio Carlos Saraiva da Costa ${ }^{2}$
}

Resumo

Magnesioferrita é um importante mineral devido a sua utilização em diferentes campos da ciência e pelo fato de que no solo, pela ação do intemperismo, pode ser fonte de nutrientes essenciais ao desenvolvimento das plantas. A sua utilização na forma pura ou associada a outros minerais só é possível através da síntese em laboratório. Este trabalho teve por objetivo sintetizar magnesioferrita e hematita a partir de magnetita-magnesiana pelo método de co-precipitação. A metodologia utilizada é uma adaptação do método de síntese de magnetita pura, substituindo parcialmente os sais solúveis de ferro por sais solúveis de magnésio na proporção de 30,0 mol\% de Fe por Mg. Para caracterização dos materiais foram utilizadas as técnicas de difração de raios-x, análise química total e susceptibilidade magnética por unidade de massa. Os resultados mostraram que além de magnetita-magnesiana foi sintetizada uma inédita muskoxita, que após o tratamento térmico foi convertida a magnesioferrita e hematita na proporção de $93,1 \%$ e $6,9 \%$ respectivamente. A substituição isomórfica do $\mathrm{Fe}$ por $\mathrm{Mg}$ aumentou a estabilidade térmica do mineral ferrimagnético sintetizado.

Palavras-chave: Óxidos de ferro. Ferrimagnetismo. Susceptibilidade magnética. Maghemita.

\begin{abstract}
Magnesioferrite is an important mineral due to its use in different scientific fields and by the fact that the soil through the action of weathering, can be a source of nutrients essential for plant development by the fact that in the soil. Its use in pure form or associated with other minerals is only possible through the synthesis in laboratory conditions. This study aimed to synthesize magnesioferrite and hematite from magnesian-magnetite by a co-precipitation procedure. The methodology used is an adaptation of the method of synthesis of pure magnetite, partially replacing the soluble salts of iron with soluble magnesium salts in the proportion of $30.0 \mathrm{~mol} \%$ of $\mathrm{Fe}$ for $\mathrm{Mg}$. The characterization of the synthetic minerals used $\mathrm{x}$-rays diffraction, total chemical analysis and mass specific magnetic susceptibility. The results showed that besides the magnesian-magnetite an unprecedented muskoxita was synthesized, which upon annealing was converted to magnesioferrite and hematite and in the proportion of $93.1 \%$ and $6.9 \%$ respectively. The isomorphous substitution of $\mathrm{Fe}$ for $\mathrm{Mg}$ enhanced the thermal stability of the ferrimagnetic mineral synthesized.
\end{abstract}

Keywords: Iron oxides. Ferrimagnetism. Magnetic susceptibility. Maghemite.

1 Doutorando em Agronomia, Universidade Estadual de Maringá; mhanami@gmail.com.

2 Professor Associado, PhD. Departamento de Agronomia, Universidade Estadual de Maringá; acscosta@uem.br. 


\section{Introdução}

O Estado do Paraná possui $50 \%$ da sua área coberta com solos que apresentam grande quantidade de magnetitas substituídas por magnésio. A magnetita é um mineral acessório em rochas magmáticas ferromagnesianas (dioritos, gabros, peridotitos e equivalentes vulcânicos) e de rochas metamórficas. Este mineral possui em sua estrutura, em substituição isomórfica ao ferro, os elementos $\mathrm{Mg}^{2+}, \mathrm{Mn}^{2+}$ e $\mathrm{Zn}^{2+}$, em proporções variadas e de forma muito comum na natureza. Devido à esta característica química, no processo de intemperismo, a magnetita se torna importante fonte de micronutrientes às plantas (MELO; CASTILHO; PINTO, 2009).

A presença do magnésio durante o processo de formação dos minerais de ferro, em substituição isomórfica, como descritos por Gaspar e Wyllie (1983), Mitchell (1978), Silva et al. (2005a) e Silva et al. (2005b), forma a magnesioferrita $\left(\mathrm{MgFe}_{2} \mathrm{O}_{4}\right)$ que no processo de intemperismo, pode ser precursora de maghemita $\left(\gamma-\mathrm{Fe}_{2} \mathrm{O}_{3}\right)$ e ou hematita $\left(\alpha-\mathrm{Fe}_{2} \mathrm{O}_{3}\right) . \mathrm{O}$ mineral magnesioferrita, além de presente nos solos, tem importância no campo de catálise heterogênea, adsorção, fabricação de sensores e tecnologias magnéticas (WILLEY; NOIRCLERC; BUSCA, 1993).

No processo de formação dos minerais secundários de ferro no solo, a liberação de Fe(II) dos minerais primários, incluindo a magnetita, pode seguir diferentes rotas. O esquema das reações químicas envolvidas na formação e evolução desses minerais em diferentes condições ambientais é representado na Figura 1.

No entanto, os trabalhos de identificação e caracterização de óxidos e hidróxidos de ferro formados em solos e sedimentos é complexo pelas dificuldades de separação e concentração destes minerais, além da grande número de variáveis que influenciam na sua formação e transformações. Assim, a síntese destes minerais em condições controladas de laboratório permite melhor controle destas variáveis, impossíveis a campo.
Figura 1 - Representação esquemática das reações químicas envolvidas na formação e transformação dos óxidos de ferro segundo variáveis ambientais. M.O. matéria orgânica.

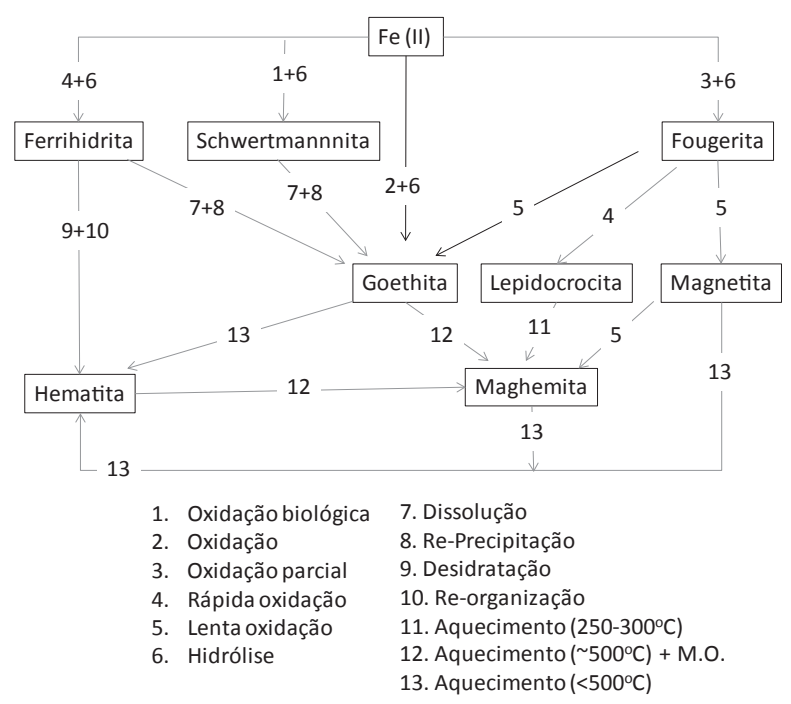

Fonte: Costa e Bigham (2009).

A síntese de hematita seguindo-se a rota magnetita $\rightarrow$ hematita, é semelhante à síntese de magnesioferrita que pode ser realizada por diferentes métodos, tendo todos em comum o aquecimento a temperaturas que variam de $80^{\circ} \mathrm{C}$ a $1260^{\circ} \mathrm{C}$.

Candeia et al. (2006), utilizaram o método solgel para sintetizar magnesioferrita que consiste, basicamente, na utilização de nitrato de ferro (III) e carbonato de magnésio além de ácido cítrico e etileno-glicol, para promover a esterificação, sendo que o final da reação é a formação de um gel polimérico. Holec et al. (2009), sintetizaram magnesioferrita através da hidrólise de microemulsão contendo solução de alkóxidos por adição de água, sendo que a solução de alkóxidos foi preparada utilizando metóxido de magnésio e etóxido de ferro em etanol. Kulkarni e Joshi (1986), Wang et al. (2006) e Nakagomi et al. (2009), utilizaram o método da reação de combustão, com a mistura de $\mathrm{MgO}$ e $\mathrm{Fe}_{2} \mathrm{O}_{3}$ em aquecimento por um tempo de até 12 horas a uma temperatura de $1260^{\circ} \mathrm{C}$ para sintetizar magnesioferrita.

De acordo com Verma et al. (2004), a síntese 
de magnesioferrita pode ser efetuada pelo método hidrotérmico em microondas, com aquecimento a $150^{\circ} \mathrm{C}$ e pressão de 50 psi por 25 minutos, em frascos de teflon.

Diversos autores (SEPELAK et al. 2007; SEPELAK et al. 2003, SEPELAK et al. 2001, SEPELAK et al. 2006; SEPELAK; BECKER, 2000; PRADHAN et al. 2011; PRADHAN; SAIN; DUTTA, 2005; PAVLOVIC et al. 2009) utilizam o método de mecanossíntese que consiste na moagem a elevadas rotações de misturas de óxidos de ferro e magnésio utilizando-se esferas de aço de elevada dureza.

O último método a citar é o que utiliza a coprecipitação (CHEN et al. 1999; HANKARE et al. 2009a; HANKARE et al. 2009b; HOQUE et al. 2011) e consiste na mistura de soluções de $\mathrm{Fe}^{2+}$ e $\mathrm{Mg}^{2+}$, em proporção molar 2:1 respectivamente, na presença de uma base forte $(\mathrm{NaOH}$ ou $\mathrm{KOH})$, com aquecimento a aproximadamente $100^{\circ} \mathrm{C}$. O precipitado formado é lavado para retirada do excesso de sais, secados em estufa e aquecidos de 400 a $900^{\circ} \mathrm{C}$ por 3 horas.

Este trabalho teve por objetivos sintetizar magnetita, magnetita-magnesiana, magnesioferrita e hematita, modificando o procedimento original de síntese de magnetitas (SCHWERTMANN; CORNELL, 2000) por co-precipitação, além de verificar a eficácia dos métodos de síntese e caracterização dos materiais produzidos.

\section{Materiais e Métodos}

\section{Síntese da magnetita}

A síntese de magnetita seguiu o método descrito por Schwertmann e Cornell (2000), e consistiu em dissolver $80 \mathrm{~g}$ de uma solução de sulfato ferroso em $560 \mathrm{~mL}$ de água deionizada com fluxo prévio de $\mathrm{N}_{2}$, em frasco de vidro com capacidade de $1,0 \mathrm{~L}$ coberta com película plástica, onde foram inseridos um termômetro e um funil dosador. O sistema ficou aquecido a $90^{\circ} \mathrm{C}$ e agitado com agitador magnético.
Quando a solução atingiu a temperatura de $90^{\circ} \mathrm{C}$ foi adicionado $240 \mathrm{~mL}$ de uma solução com água deionizada e livre de oxigênio contendo 6,46 g de $\mathrm{KNO}_{3}$ e 44,9 g $\mathrm{KOH}$ em aproximadamente 5 minutos.

O sistema permaneceu 120 minutos sob aquecimento, e após foi deixado em repouso durante uma noite. Logo depois desse processo o precipitado negro formado foi lavado com água deionizada até que a condutividade elétrica fosse menor do que 0,2 $\mathrm{mS} \mathrm{m}^{-1}$. Após a síntese do mineral não foi necessário manter o mineral formado isolado do contato com o ar devido a sua estabilidade.

\section{Sintese da magnetita magnesiana}

A produção de magnetitas com substituição isomórfica do ferro por magnésio seguiu procedimento semelhante ao da magnetita pura com a adição de sais solúveis de magnésio, no início da reação e antes da adição da solução contendo $\mathrm{KOH}$. Após a co-precipitação, os materiais sólidos foram lavados em água deionizada para remoção do excesso de sais, congelados em nitrogênio líquido e liofilizados. Os materiais sintetizados e liofilizados analisados nos seus atributos físicos, químicos e mineralógicos.

A proporção de magnésio substituindo ferro utilizada na síntese da magnetita magnesiana foi de 30,0 mol\%, isto é, a proporção nominal de x na fórmula química $\mathrm{Fe}_{3-\mathrm{x}} \mathrm{Mg}_{\mathrm{x}} \mathrm{O}_{4}$ foi de 0,90 .

\section{Sintese da magnesioferrita}

Parte do material sintetizado na etapa de obtenção da magnetita magnesiana foi aquecido a $800^{\circ} \mathrm{C}$ por 4 horas para conversão de magnetitas-magnesianas em magnesioferrita.

\section{Análise química dos materiais}

Duplicatas do material sintetizados na forma de pó (aproximadamente $100 \mathrm{mg}$ ) foram colocadas em 
tubos de vidro borossilicato com $15 \mathrm{~mL}$ de $\mathrm{H}_{2} \mathrm{SO}_{4}$ $(20 \% \mathrm{v} / \mathrm{v})$, e colocados em bloco digestor a uma temperatura constante de $350^{\circ} \mathrm{C}$ até a dissolução total. A solução foi guardada e os conteúdos de Fe e $\mathrm{Mg}$ foram determinados por espectrofotometria de absorção atômica. Os teores de $\mathrm{Mg}$ e Fe teórico e experimental foram calculados em função das quantidades utilizadas na síntese em comparação com os valores obtidos na análise química (EMBRAPA, 1987).

\section{Difração de raios- $X$}

O material sintetizado em pó foi analisado em um difratômetro de raios $X$, equipamento Shimadzu XRD 6000 utilizando radiação $\mathrm{CuK \alpha}$, com monocromador de grafite, modo passo $(0,02$ ${ }^{\circ} 2 \theta, 2 \mathrm{~s}$ ), conforme Gardes et al., (2009), com adaptações. Os resultados foram exportados para software GRAMS 8® para a determinação das áreas e das posições dos reflexos, da largura a meia altura e posteriormente calculadas os diâmetros médios cristalinos (DMC) utilizando a equação de Scherrer (KLUG; ALEXANDER, 1974). Para a magnetita pura foi adicionado $(\sim 5 \% \mathrm{~g} / \mathrm{g})$ padrão interno de silício ( $\mathrm{Si}$ ) para possível correção de deslocamento dos reflexos característicos, o que não foi feito para os materiais sintetizados com substituição isomórfica do ferro por magnésio.

A participação da magnesioferrita (Mf) e hematita $(\mathrm{Hm})$ em relação ao total dos óxidos de $\mathrm{Fe}$ foi determinada utilizando-se as áreas dos reflexos referentes aos planos de reflexão (220) e (012), respectivamente (COSTA et al., 1999). Para o cálculo da percentagem das fases cristalinas formadas, a área referente ao plano (220) da magnesioferrita foi multiplicada por 2,5, a área referente ao plano (012) da hematita foi também multiplicada por 3,5. O cálculo foi, então, realizado utilizando as proporções a partir das equações:
$\operatorname{Mf}(\%)=3,5 \times$ Área $M f d_{220} /\left(3,5 \times\right.$ Área $M f d_{220}+$ 3,5 x Área $\mathrm{Hm} \mathrm{d}_{012}$ ) x 100

$\operatorname{Hm}(\%)=3,5 \times$ Area $\operatorname{Hm~d}_{012} /\left(3,5 \times\right.$ Area $\mathrm{Mf} \mathrm{d}_{220}$ $+3,5 \times$ Area $\left.\mathrm{Hm} \mathrm{d}_{012}\right) \times 100$

Suscetibilidade magnética $\left(\chi_{B F}\right)$ por unidade de massa dos materiais

Para determinação da susceptibilidade magnética por unidade de massa $(\chi)$ foi utilizada uma massa de aproximadamente $150 \mathrm{mg}$ de amostra do material sintetizado em recipientes plásticos de $10 \mathrm{~cm}^{3} \mathrm{e}$ completado este volume com açúcar, material inerte magneticamente. A susceptibilidade magnética por unidade de massa foi determinada utilizandose um sistema Bartington MS2 (Bartington Instruments LTD, Oxford, Inglaterra) acoplado a um sensor MS2B. A sensibilidade deste sensor é elevada podendo detectar em pequenas quantidades elementos ferrimagnéticos (DEARING, 1994). A susceptibilidade magnética por unidade de massa foi determinada em baixa freqüência $\left(\chi_{\mathrm{BF}}\right)$, utilizando os valores da massa da amostra (m) e da susceptibilidade magnética volumétrica $(\kappa)$ partir da equação:

$$
\chi_{\mathrm{BF}}=\left(10 \kappa \mathrm{m}^{-1}\right)
$$

onde $\kappa=$ adimensional.

\section{Resultados e Discussão}

Os resultados da análise química total (Tabela 1) mostram que o magnésio adicionado substituiu o ferro na estrutura mineral dos materiais sintetizados. A percentagem de substituição isomórfica do Fe pelo $\mathrm{Mg}$ variou de $28 \%$ nos sólidos antes do aquecimento e $30 \%$ após aquecimento de $800^{\circ} \mathrm{C}$ por 4 horas. Isto é, independente das formas minerais coprecipitadas, $\mathrm{Mg}$ foi inserido na estrutura cristalina das formas de ferro. 
Os resultados de difração de raios-X mostram que além da magnetita-magnesiana, formou-se também muskoxita que é um óxido de ferro magnesiano hidratado com cela unitária trigonal. Jambor (1969) descreveu este novo mineral, a muskoxita, encontrado na região noroeste do Canadá, que apresenta fórmula química $\left[\mathrm{Mg}_{3,41} \mathrm{Fe}_{2} \mathrm{O}_{6,41} .5,1 \mathrm{H}_{2} \mathrm{O}\right]$, ou ainda na forma de óxidos $3,41 \mathrm{MgO}$. $\mathrm{Fe}_{2} \mathrm{O}_{3} \cdot 5,1 \mathrm{H}_{2} \mathrm{O}$. A presença da muskoxita associada à magnetita-magnesiana influenciou na pequena redução do valor obtido de SI. Após o aquecimento a $800^{\circ} \mathrm{C} / 4$ horas este efeito desapareceu e observouse um aumento do grau de SI devido a desidratação e à transformação da muskoxita em magnesioferrita.

Tabela 1 - Substituição isomórfica calculada em função dos resultados da análise química.

\begin{tabular}{lcc}
\hline Mineral & \multicolumn{2}{c}{ SI (mol\%) } \\
& Teórica & Experimental \\
\hline Magnetita & 0 & 0 \\
Magnetita-Mg & 30 & 28,21 \\
Magnetita-FM & 30 & 30,34 \\
\hline
\end{tabular}

Fonte: Os autores.

\section{Difração de Raios- $X$}

Os resultados de difração de raios-x são apresentados nas figuras 2, 3 e 4 .

As intensidades dos reflexos da magnetita sintética pura coincidem com o padrão do cartão 88-0315 (JOINT COMMITTEE ON POWDER DIFFRACTION STANDARDS - JCPDS, 2003), conforme mostrado na figura 2. A simples observação das larguras dos reflexos mostra que a magnetita sintetizada apresenta elevado grau de cristalinidade.
Figura 2 - Difratograma de raios-X de magnetita pura sem substituição isomórfica com padrão interno silício.

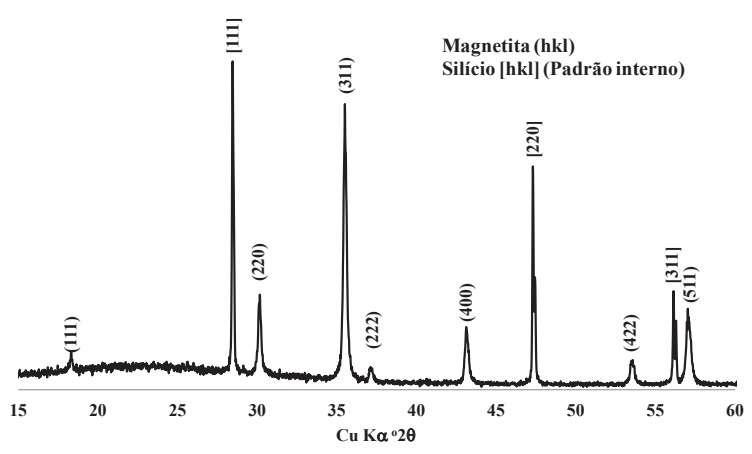

Fonte: Os autores.

A figura 3 mostra os minerais formados no processo de co-precipitação na presenga de magnésio. Além da magnetita magnesiana ocorreu ainda a formação da muskoxita (JAMBOR, 1969). Este último mineral pode ser indexado pelos reflexos $\mathrm{d}_{101}, \mathrm{~d}_{100}, \mathrm{~d}_{001}$ e $\mathrm{d}_{102}$ definidos pelo cartão 22-0709 (JCPDS, 2003). A muskoxita formada apresenta baixo grau de cristalinidade evidenciado pelos largos reflexos da figura 2. Já a magnetitamagnesiana apresentou grau de cristalinidade semelhante ao da magnetita pura e pouco diferentes dos reflexos do Si (Figura 2), utilizado como padrão interno.

Figura 3 - DRX de magnetita-magnesiana e muskoxita, antes do aquecimento.

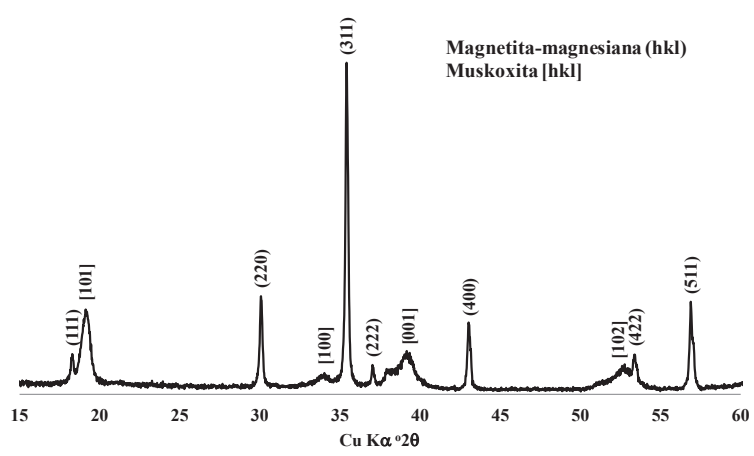

Fonte: Os autores. 
A figura 4 mostra os óxidos de ferro formados no processo de aquecimento da magnetitamagnesiana aquecida a $800{ }^{\circ} \mathrm{C}$ por 4 horas. Nestas condições foram detectados os reflexos dos minerais magnesioferrita e hematita, confirmados pelos cartões 36-0398 e 33-0664 (JCPDS, 2003), respectivamente.

Figura 4 - Difratograma de raios- $\mathrm{X}$ de magnetitamagnesiana após aquecimento a $800^{\circ} \mathrm{C}$ por 4 horas.

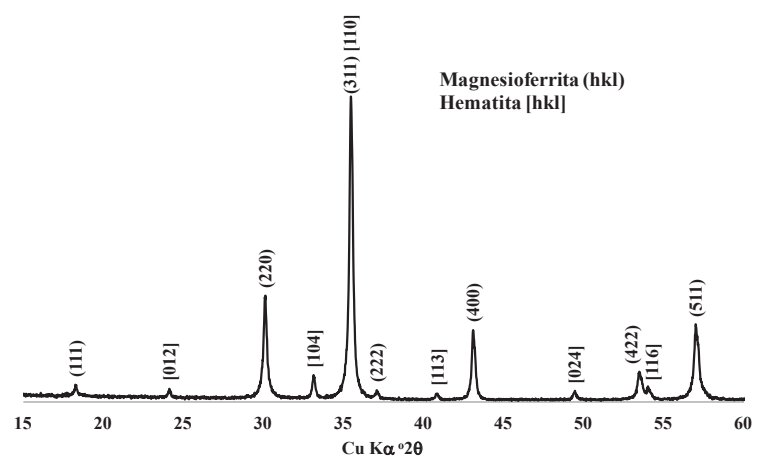

Fonte: Os autores.

Os parâmetros de rede dos minerais sintetizados em comparação com os minerais padrões do sistema cúbico são apresentados na tabela 2. Os valores obtidos de tamanho (a $\mathrm{a}_{\mathrm{o}}$ e de volume (Vol.) da cela unitária nos materiais sintetizados são muito próximos dos valores de minerais padrões (JCPDS, 2003). O pequeno aumento no tamanho da cela unitária pode estar relacionado à ocupação pelo $\mathrm{Mg}$ dos sítios tetraedrais e octaedrais onde estão alocados $\mathrm{Fe}^{3+}$ e $\mathrm{Fe}^{2+}$ respectivamente. Neste caso o $\mathrm{Mg}^{2+}$ está substituindo parcialmente o $\mathrm{Fe}^{3+}$ visto que estes cátions têm raios iônicos de $0,072 \mathrm{~nm}$ e $0,065 \mathrm{~nm}$, respectivamente. Estes resultados são confirmados por Sepelak et al. (2003), que encontraram para este mineral a fórmula química $\left(\mathrm{Mg}_{0,24} \mathrm{Fe}_{0,76}\right)^{\mathrm{T}}\left[\mathrm{Mg}_{0,76} \mathrm{Fe}_{1,24}\right]^{\mathrm{O}} \mathrm{O}_{4}$, onde os elementos entre parênteses $(\mathrm{T})$ representa o sítio tetraedral e entre colchetes $[\mathrm{O}]$ o sítio octaedral.
Tabela 2 - Parâmetros de rede dos minerais formados nas sínteses.

\begin{tabular}{lccc}
\hline Minerais & $\begin{array}{c}\mathrm{a}_{0} \\
(\mathrm{~nm})\end{array}$ & $\begin{array}{c}\mathrm{DMC} \\
\left(\mathrm{nm}^{3}\right)\end{array}$ \\
\hline Magnetita Padrão & 0,8375 & - & 0,587 \\
Magnetita Sintetizada & 0,8382 & 39,78 & 0,59 \\
Magnesioferrita Padrão & 0,8387 & - & 0,59 \\
Magnesioferrita Sintetizada & 0,8393 & 30,22 & 0,591 \\
\hline
\end{tabular}

DMC-diâmetro médio cristalino, Vol-volume.

Fonte: Os autores.

Com os dados obtidos nos difratogramas de raios-X e utilizando-se o programa GRAMS $\AA 8.0$ foi possível calcular a proporção de cada mineral formado após o aquecimento a $800^{\circ} \mathrm{C} / 4$ horas (Tabela 3).

Tabela 3 - Proporção dos minerais sintetizados calculados com base no programa GRAMS8 ${ }^{\circledR}$ na amostra de magnetita magnesiana após aquecimento a $800^{\circ} \mathrm{C}$ por 4 horas.

\begin{tabular}{lc}
\hline Mineral & $(\%)$ \\
\hline Magnesioferrita & 93,1 \\
Hematita & 6,9 \\
\hline
\end{tabular}

Fonte: Os autores.

A maior parte da magnetita magnesiana formou magnesioferrita (93,1\%). Além deste mineral também foi identificada e quantificada o mineral hematita $(6,9 \%)$. A formação da hematita pode ser creditada à presença de óxidos hidratados de ferro pobremente cristalinos (Ex. Ferrihidrita) que não podem ser observados na Figura 3 devido a sai baixa concentração $(<10 \%)$ e baixo grau de cristalinidade. No entanto estes minerais, em temperaturas próximas a $500^{\circ} \mathrm{C}$ perdem a água de constituição formando hematita (COSTA; BIGHAM, 2009). Ainda, é possível concluir que toda muskoxita foi desidratada e formou magnesioferrita. Portanto, a muskoxita pode ser considerada um mineral 
precursor da magnesioferrita quando aquecida a altas temperaturas. A estequiometria da reação poderia descrita como:

$$
\mathrm{Mg}_{7} \mathrm{Fe}_{4} \mathrm{O}_{13} \cdot 10 \mathrm{H}_{2} \mathrm{O} \rightarrow 2 \mathrm{Fe}_{2} \mathrm{MgO}_{4}+5 \mathrm{MgO}+10 \mathrm{H}_{2} \mathrm{O}
$$

A presença de $\mathrm{MgO}$ (Periclase) não observada nos difratogramas de raios-X (Figura 4) é um indício de que: 1. Parte do $\mathrm{Mg}^{2+}$ pode ter substituído o ferro na estrutura da hematita, ou 2. Sua concentração é inferior a 5\%, difícil de ser detectado por difração de raios-X no modo passo $0,02^{\circ} 3 \theta / 0,6 \mathrm{~s}$. Estas hipóteses serão verificadas em experimentos subsequentes.

Susceptibilidade magnética por unidade de massa $\left(\chi_{\mathrm{BF}}\right)$

Os valores de susceptibilidade por unidade de massa confirmam os dados difração de raios-X com a formação da magnetita pura (Figura 2), magnetita magnesiana (Figura 3) e que a substituição do ferro pelo magnésio na magnesioferrita reduz o magnetismo do mineral (Figuras 3 e 4, Tabela 4).

Os resultados de $\chi_{\mathrm{BF}}$ estão na faixa de variação observada para minerais ferrimagnéticos enumerados por Costa e Bigham (2009), que atribuem aos minerais ferrimagnéticos valores de $\chi_{\mathrm{BF}}$ que variam numa faixa de 16.900 (Titanomagnetitas) a $111.600 \times 10^{-8} \mathrm{~m}^{3} \mathrm{~kg}^{-1}$ (Magnetitas). A presença do magnésio, um elemento diamagnético em substituição isomórfica ao ferro, reduziu os valores de susceptibilidade magnética em 60\% (Tabela 4) (BATISTA et al., 2008). Com o aquecimento da amostra a $800^{\circ} \mathrm{C} / 4 \mathrm{~h}$ os valores de $\chi_{\mathrm{BF}}$ foram ainda menores, cerca de $38 \%$ do valor da magnetita pura. A redução no valor de $\chi_{\mathrm{BF}}$ entre a magnetita magnesiana e a magnesioferrita é de $6,5 \%$, muito próximo do valor calculado $(6,9 \%)$ da percentagem da hematita obtida por DRX. A hematita é um mineral antiferromagnético (COSTA; BIGHAM, 2009) e sua presença na amostra diluição os valores de $\chi_{\mathrm{B}}$, reduzindo o valor final da amostra.

Tabela 4 - Susceptibilidade magnética $\left(\chi_{\mathrm{BF}}\right)$ por unidade de massa dos minerais de ferro sintetizados por coprecipitação.

\begin{tabular}{lc}
\hline Mineral & $\begin{array}{c}\chi_{\mathrm{BF}} \\
10^{-8} \mathrm{~m}^{3} \mathrm{~kg}^{-1}\end{array}$ \\
\hline Magnetita pura & 48025 \\
Magnetita-magnesiana & 19558 \\
Magnesioferrita & 18280 \\
\hline
\end{tabular}

Fonte: Os autores.

\section{Conclusões}

A magnetita magnesiana sintética apresentou $28 \%$ de substituição isomórfica de $\mathrm{Fe}$ por $\mathrm{Mg}$, enquanto a magnesioferrita apresentou $30 \%$ de SI.

A síntese de magnetita magnesiana resultou também na formação de muskoxita. $\mathrm{O}$ aquecimento desta amostra a $800^{\circ} \mathrm{C}$ por 4 horas formou magnesioferrita e hematita.

A substituição isomórfica do Fe por $\mathrm{Mg}$ reduziu os valores de $\chi_{\mathrm{BF}}$ em $60 \%$ em relação a magnetita pura e $272 \%$ após aquecimento a $800^{\circ} \mathrm{C}$ por 4 horas.

A substituição isomórfica do $\mathrm{Fe}$ pelo $\mathrm{Mg}$ aumentou a estabilidade do mineral ferrimagnético formado impedindo sua transformação para hematita e mantendo parte de seus atributos magnéticos mesmo após aquecimento a $800^{\circ} \mathrm{C}$.

\section{Agradecimentos}

Os autores agradecem ao Complexo de Centrais de Apoio à Pesquisa (COMCAP), onde foram realizadas as análises de difratometria de raios-X. 


\section{Referências}

BATISTA, M. A.; COSTA, A. C. S.; SOUZA JUNIOR, I. G.; BIGHAM, J. M. Cristallochemical characterization of synthetic $\mathrm{Zn}$-substituted maghemite $\left(\gamma-\mathrm{Fe}_{2}-\mathrm{xZn}_{\mathrm{x}} \mathrm{O}_{3}\right)$. Revista Brasileira de Ciência do Solo, Viçosa, v. 32, p. 561-568, 2008.

CANDEIA, R. A.; SOUZA, M. A. F.; BERNARDI, M. I. B.; MAESTRELLI, S. C.; SANTOS, I. M. G.; SOUZA, A. G.; LONGO, E. $\mathrm{MgFe}_{2} \mathrm{O}_{4}$ pigment obtained at low temperature. Materials Research Bulletin, New York, v. 41, p. 183-190, 2006.

CHEN, Q.; RONDINONE, A. J.; CHAKOUMAKOS, B. C.; ZHANG, Z. J. Synthesis of superparamagnetic $\mathrm{MgFe}_{2} \mathrm{O}_{4}$ nanoparticles by coprecipitation. Journal of Magnetism and Magnetic Materials, Amsterdam, v. 194, p. 1-7, 1999.

COSTA, A. C. S.; BIGHAM, J. M. Óxidos de ferro. In: MELO, V. F.; ALLEONI, R. F. (Ed.). Quimica e mineralogia do solo, , Viçosa: SBCS, 2009. Parte 1.

COSTA, A. C. S.; BIGHAM, J. M.; RHOTON, F. E.; TRAINA, S. J. Quantification and characterization of maghemite in soils derived from volcanic rocks in southern Brazil. Clays and Clay Minerals, New York, v. 47, n. 4, p. 466-473, 1999.

DEARING, J. Environmental magnetic susceptibility: using Bartington MS2 system. Kenilworth: Chi Publishing, 1994.

EMPRESA BRASILEIRA DE PESQUISA AGROPECUÁRIA. Centro Nacional de Pesquisa de Solos. Manual de métodos de análise de solos. Rio de Janeiro: EMBRAPA, 1987. (EMBRAPA-CNPS. Documentos, v. 1).

GARDES, B. J. L.; ALFAYA, A. A. S.; ALFAYA, R. V. S.; URBANO, A.; SCARMÍNIO, J. Preparação de $\mathrm{LiMn}_{2} \mathrm{O}_{4}$ e $\mathrm{LiCr}_{0,2} \mathrm{Mn}_{1,8} \mathrm{O}_{4}$ em pó por processo Pechini modificado. Semina: Ciências Exatas e Tecnológicas, Londrina, v. 30, n. 2, p. 91-98, jul./dez. 2009.

GASPAR, J. C.; WYLLIE, P. J. Magnetite in the carbonatites from the jacupiranga complex, Brazil. American Mineralogist, Washington, v. 68, p. 195-213, 1983.

HANKARE, P. P.; JADHAV, S. D.; SANKPAL, U. B.; PATILA, R. P.; SASIKALAB, R.; MULLAC, I. S. Gas sensing properties of magnesium ferrite prepared by coprecipitation method. Journal of Alloys and Compounds, Lausanne, v. 488, n. 1, p. 270-272, 2009a.

HANKARE, P. P.; VADER, V. T.; PATIL, N. M.; JADHAV, S. D.; SANKPAL, U. B.; KADAM, M. R.; CHOUGUle, B. K.; GAJBHIYE, N. S. Synthesis, characterization and studies on magnetic and electrical properties of $\mathrm{Mg}$ ferrite with $\mathrm{Cr}$ substitution. Materials Chemistry and Physics, Lausanne, v. 113, n. 1, p. 233238, 2009b.

HOLEC, P.; PLOCEK, J.; NIŽŇANSKÝ, D.; POLTIEROVÁ VEJPRAVOVÁ, J. Preparation of $\mathrm{MgFe}_{2} \mathrm{O}_{4}$ nanoparticles by microemulsion method and their characterization. Journal of sol-gel science and technology, Dordrecht, n. 51, p. 301-305, 2009.

HOQUE, S. M.; HAKIM, M.A.; MAMUN, A.; AKHTER, S.; HASAN, T.; PAUL, D. P.; CHATTOPADHAYAY, K. Study of the bulk magnetic and electrical properties of $\mathrm{MgFe}_{2} \mathrm{O}_{4}$ synthesized by chemical method. Materials Sciences and Applications, Dover, v. 2, n. 11, p. 15641571, 2011.

JAMBOR, J. L. Muskoxite, a new hydrous magnesiumferric iron oxide from the muskox intrusion, northwest territories, Canada. American Mineralogist, Washington, v. 54, p. 684-696, May-Jun. 1969.

JOINT COMMITTEE ON POWDER DIFFRACTION STANDARDS - JCPDS. International Center for Diffraction Data, 22-0709, 33-0664, 36-0398, 88-0315, 2003.

KLUG, H. P.; ALEXANDER, L. E. X-ray diffraction procedures for polycrystalline and amorphous materials. 2. ed. New York: Willey, 1974.

KULKARNI, R. G.; JOSHI, H. H. Comparison of magnetic properties of $\mathrm{MgFe}_{2} 0_{4}$ prepared by wetchemical and ceramic methods. Journal Of Solid State Chemistry, San Diego, n. 64, p. 141-147, 1986.

MELO, V. F.; CASTILHOS, R. M. V.; PINTO, L. F. S. Reserva mineral do solo. In: MELO, V. F.; ALLEONI, R. F. (Ed.). Quimica e mineralogia do solo. Viçosa: SBCS, 2009. Parte 1.

MITCHELL, R. H. Manganoan magnesian ilmenite and titanian clinohumite from the Jacupiranga carbonatite, São Paulo. Brazil. American Mineralogist, Washington, v. 63 , p. $544-547,1978$.

NAKAGOMI, F.; SILVA, S. W.; GARG, V. K.; OLIVEIRA, A. C.; MORAIS, P. C.; FRANCO, A. Influence of the Mg-content on the cation distribution in cubic $\mathrm{MgxFe}_{3 \times} \mathrm{O}_{4}$ nanoparticles. Journal of Solid State Chemistry, San Diego, n. 182, p. 2423-2429, 2009.

PAVLOVIC, M.; JOVALEKIĆ, Č.; NIKOLIĆ, A. S.; MANOJLOVIĆ, D.; ŠOJIĆ, N. Mechanochemical synthesis of stoichiometric $\mathrm{MgFe}_{2} \mathrm{O}_{4}$ spinel. Journal of Materials Science: Materials in Electronics, London, v. 20, p. 782-787, 2009. 
PRADHAN, S.; BID, S.; GATESHKI, M.; PETKOV, V. Microstructure characterization and cation distribution of nanocrystalline magnesium ferrite prepared by ball milling. Materials Chemistry and Physics, Lausanne, v. 93, p. 224-230, 2005.

PRADHAN, S. K.; SAIN, S.; DUTTA, H. Microstructure characterization of nanocrystalline magnesium ferrite annealed at elevated temperatures by Rietveld method. ISRN Ceramics, New York, v. 2011, p. 1-8, 2011.

SCHWERTMANN, U.; CORNELL, R. M. Iron oxides in laboratory: preparation and characterization. Weinheim: WILEY-VCH, 2000.

SEPELAK V.; FELDHOFF, A.; HEITJANS, P.; KRUMEICH，F.; MENZEL，D.; LITTERST，F. J.; BERGMANN, I.; BECKER, K. D. Nonequilibrium cation distribution, canted spin arrangement, and enhanced magnetization in nanosized $\mathrm{MgFe}_{2} \mathrm{O}_{4}$ prepared by a one-step mechanochemical route. Chemistry of Materials, Washington, v. 18, p. 3057-3067. 2006.

SEPELAK, V.; BAABE, D.; MIENERT, D.; LITTERST, F. J.; BECKER, K. D. Enhanced magnetisation in nanocrystalline high-energy milled $\mathrm{MgFe}_{2} \mathrm{O}_{4}$. Scripta Materialia, Bethesda, v. 48, p. 961-966, 2003.

SEPELAK, V.; BECKER, K. D. Mössbauer studies in the mechanochemistry of spinel ferrites, Journal of Materials Synthesis and Processing, New York, v. 8, n. 3, p. 155-166, 2000.

SEPELAK, V.; BERGMANN, I.; MENZEL, D.; FELDHOFF, A.; HEITJANS, P.; LITTERST, F. J.; BECKER, K. D. Magnetization enhancement in nanosized $\mathrm{MgFe}_{2} \mathrm{O}_{4}$ prepared by mechanosynthesis. Journal of Magnetism and Magnetic Materials, Amsterdam, v. 316, p. 764-767, 2007.
SEPELAK, V.; SCHULTZE, D.; KRUMEICH, F.; STEINIKE, U.; BECKER, K. D. Mechanically induced cation redistribution in magnesium ferrite and its thermal stability. Solid State Ionics, Amsterdam, v. 141-142, p. 677-682, 2001.

SILVA, F. D.; COUCEIRO, P. R. C.; FABRIS, J. D.; GOULART, A. T.; KER; J. C. Magnesioferrita e caminho pedogenético de transformação de óxidos de ferro magnéticos em dois perfis de solo derivados de tufito da região do Alto Paranaíba (MG). Revista Brasileira de Ciência do Solo, Viçosa, v. 29, p. 763-775, 2005a.

Óxidos de ferro magnéticos de um tufito da região do Alto Paranaíba, MG. Química Nova, São Paulo, v. 28, n. 1, p. $5-9,2005$ b.

VERMA, S.; JOY, P. A.; KHOLLAM, Y. B.; POTDAR, H. S.; DESHPANDE, S. B. Synthesis of nanosized $\mathrm{MgFe}_{2} \mathrm{O}_{4}$ powders by microwave hydrothermal method. Materials Letters, Amsterdam, v. 58, p. 1092-1095, 2004.

WANG, Z.; LAZORB, P.; SAXENA, S. K.; O'NEILL, H. St. C. High pressure Raman spectroscopy of ferrite $\mathrm{MgFe}_{2} \mathrm{O}_{4}$. Materials Research Bulletin, New York, v. 37, p. 1589-1602, 2006.

WILLEY, R. J.; NOIRCLERC, P. BUSCA, G. Preparation and chacterization of magnesium cromite and magnesium ferrite aerogels. Chemical Engineering Communications, New York, v. 123, p. 1-16, 1993. 
